\title{
Evaluate the inhibition of cytochrome P450 1A1 for enhancing breast cancer chemotherapy with a turn-on fluorescent probe
}

\author{
Yan Huang ${ }^{\mathrm{a}, *}$, Lei $\mathrm{Yu}^{\mathrm{b}}$, Pengpeng Lu ${ }^{\mathrm{a}}$, Yinghui Wei ${ }^{\mathrm{d}}$, Xiaoyan Wang ${ }^{\mathrm{a}, \mathrm{c}}$, Lingxin Chen ${ }^{\mathrm{a}, \mathrm{c}, \mathrm{e}, *}$ \\ ${ }^{a}$ School of Pharmacy, Binzhou Medical University, Yantai, 264003, China \\ ${ }^{\mathrm{b}}$ Shandong Peninsula Engineering Research Center of Comprehensive Brine Utilization, Weifang University of Science and Technology, Weifang, 262700, China \\ ${ }^{\mathrm{c}}$ CAS Key Laboratory of Coastal Environmental Processes and Ecological Remediation, Shandong Key Laboratory of Coastal Environmental Processes, Yantai Institute of \\ Coastal Zone Research, Chinese Academy of Sciences, Yantai, 264003, China \\ d Department of Respiratory Medicine, Binzhou Medical University Hospital, Binzhou, 256603, China \\ ${ }^{\mathrm{e}}$ College of Chemistry and Chemical Engineering, Qufu Normal University, Qufu, 273165, China
}

\section{A R T I C L E I N F O}

\section{Keywords:}

Fluorescent probe

Cytochrome P450 1A1

Chemotherapy

Breast cancer

Fluorescence imaging

\begin{abstract}
A B S T R A C T
The incidence rate of breast cancer ranks first in women malignant tumors worldwide, accompanied by a high mortality. The early diagnosis and therapy are important way to reduce the mortality rate of breast cancer in clinical practice. There are urgent needs for anti-tumor strategies with clear evidence and reliable efficacy. Because cytochrome P450 1A1 (CYP1A1) is commonly expressed in breast cancer and has the excellent ability to metabolize xenobiotics, researchers are interested in studying the vital function of CYP1A1 in breast cancer. Herein, we report a BCy-CYP fluorescent probe for imaging of CYP1A1 in cells and in vivo. This probe has outstanding selectivity and high sensitivity for CYP1A1 detection over other biomolecules. Our results reveal that the intracellular CYP1A1 level increase in MCF-7 and MDA-MB-231 cells, and inhibition of CYP1A1 by carnosol efficiently induce apoptosis in the two kinds of breast cancer cells. Thus, CYP1A1 plays a crucial role in breast cancer. The synergistic effect of carnosol and chemotherapy drug is better than chemotherapy drug alone in tumor-bearing mice. CYP1A1 level may be a new biomarker for breast cancer early diagnosis.
\end{abstract}

\section{Introduction}

Breast cancer is one of the most common malignant tumors in women worldwide, accompanied by the high morbidity and mortality. According to statistics, 630,000 patients died of breast cancer every year, accounting for approximately $14 \%$ of the total number of tumorrelated deaths among women worldwide [1]. Currently, the standard clinical treatments for breast cancer include surgery, chemotherapy, radiotherapy, and immunotherapy [2]. Breast cancers classification based on the expression of ER/PR and HER2 are significant as they represent different clinical and biological outcomes [3]. The most aggressive subtype is triple negative breast cancer (TNBC) that exhibit decreased survival, because no receptor (ER-, PR-, HER2-) is present on these breast tumors. TNBC accounts for approximately $15 \%$ of all breast cancer diagnoses and lacks recognized therapeutic targets. To date, cytotoxic chemotherapy is still the only choose of systemic treatment. Therefore, it is essential to establish a safe and effective treatment for TNBC [4]. Moreover, although hormone therapy has been proven successful in receptor positive breast tumors (ER+, PR+), relapsed patients often develop resistance to hormonal therapy, leaving these patients with no efficacious treatment options [4]. In this research, we select triple-negative and ER + breast cancers as the study subjects, which most need to improve treatment strategies.

Cytochrome P450 1A1 (CYP1A1), one of the three subtypes of the CYP1 family, is an extrahepatic phase-I enzyme which metabolizes a large number of endogenous and xenobiotic substrates [5]. The recent studies have indicated that CYP1A1 is constitutively expressed in breast tumors [6]. Vinothini has reported the expression levels of multiple xenobiotic-metabolizing enzymes, involving CYP1A1, in 60 cases of breast tumors from newly diagnosed people who had not received adjuvant therapy before. Their results revealed that CYP1A1 levels increased in breast tumor tissues compared to adjacent normal breast tissue [7]. Since CYP1A1 is ubiquitously expressed in breast cancer and has the ability to metabolize different xenobiotics, more and more interests have been displayed to exploit CYP1A1 biological activity for the diagnosis and therapy of breast cancer [8,9]. Therefore, better

\footnotetext{
* Corresponding authors at: School of Pharmacy, Binzhou Medical University, Yantai, 264003, China.

E-mail addresses: yhuang@yic.ac.cn (Y. Huang), lxchen@yic.ac.cn (L. Chen).
} 
understanding the relationship between CYP1A1 and breast cancer will improve existing treatment strategies and facilitate the development of targeted therapy.

In recent years, scientific researchers have put substantial effort into exploring effective methods to detect CYP1A1. Compared with other reported methods $[10,11]$, fluorescence bioimaging technology has the advantages of real-time monitoring, less damage to biological samples and visual detection. Small-molecule fluorescent probes, has been extensively used as a powerful and indispensably tool to monitor the biomolecules and their metabolic processes in complex living systems, owing to its a variety of advantages in non-destructiveness, effectiveness, high sensitivity, good membrane permeability and high selectivity, as well as rapid and in-situ detection [12-17]. Small-molecule fluorescent probes have versatile chemical structural modifications, making it easier to capture a variety of biological targets. This also allows for conceiving the specific fluorescent probe for a certain enzyme [18-23]. Although CYP1A1 plays a significant role in biological systems, there are limited fluorescent probes for rapid and effective detection of CYP1A1 level in vivo, the comparison of some probe of recent reports was shown in Table S1 in supporting information [24-29].

In present study, we designed a new fluorescent probe BCy-CYP to test the level of CYP1A1 in living cells and in vivo. BCy-CYP probe has a specific CYP1A1 substrate, it has high sensitivity toward CYP1A1 in living systems. Herein, we successfully employ BCy-CYP to monitor CYP1A1 level in MCF-7 and MDA-MB-231 cell lines and their corresponding tumor-bearing mice models. Our results illustrated that the level of CYP1A1 was increased in breast cancer cells. Based on this result, BCy-CYP is the effective small-molecule fluorescent probe for monitoring CYP1A1. The date providing a direct and significant evidence on the correlation between the CYP1A1 and breast cancer in living cells. We further validated that inhibition of CYP1A1 by carnosol efficiently induced cell apoptosis. And the synergy of carnosol and cisplatin had more effectively therapeutic than the treatment with cisplatin alone. To inhibit CYP1A1 has the enormous potential in the treatment of breast cancer for synergistic chemotherapy drug administration. Therefore, BCy-CYP probe may be a valuable tool for early clinical diagnosis and therapy of breast cancer.

\section{Experimental methods}

\subsection{Synthetic procedures of $B C y-C Y P$}

4-(Bromomethyl) benzaldehyde ( $0.2 \mathrm{~g}, 1 \mathrm{mmol})$ and Compound a ( $0.365 \mathrm{~g}, 1 \mathrm{mmol})$ were dissolved in ethanol. The mixture was stirred under reflux at $80^{\circ} \mathrm{C}$. After $48 \mathrm{~h}$, the mixture was concentrated under vacuum. The crude product was purified by silica gel chromatography with $\mathrm{CH}_{2} \mathrm{Cl}_{2} / \mathrm{CH}_{3} \mathrm{OH}(10: 1$, v/v) as eluent. Finally, BCy-CYP $(0.242 \mathrm{~g}, 63$ $\%$ yield) was obtained (a yellow product). ${ }^{1} \mathrm{H}$ NMR (500 MHz, $\mathrm{CD}_{3} \mathrm{OD}-$ $\left.\mathrm{D}_{4}\right) \delta(\mathrm{ppm}): 8.56(\mathrm{~s}, 1 \mathrm{H}), 8.40-8.38(\mathrm{~d}, 1 \mathrm{H}), 8.24-8.21(\mathrm{~m}, 3 \mathrm{H})$, 8.13-8.11 (m, $1 \mathrm{H}), 8.03-8.01(\mathrm{~m}, 1 \mathrm{H}), 7.80-7.77(\mathrm{~m}, 1 \mathrm{H})$, 7.69-7.66 (m, 2 H), 7.56-7.53 (m, $2 \mathrm{H}), 4.94-4.90$ (m, $2 \mathrm{H}), 4.57$ (s, 2 $\mathrm{H}), 3.61-3.56(\mathrm{~m}, 2 \mathrm{H}), 2.07(\mathrm{~s}, 6 \mathrm{H}), 1.67-1.64(\mathrm{t}, 3 \mathrm{H}), 1.25-1.22(\mathrm{~m}$, $3 \mathrm{H}) .{ }^{13} \mathrm{C}$ NMR (125 MHz, CD $\left.\mathrm{OD}_{3} \mathrm{D}_{4}\right) \delta(\mathrm{ppm}): 182.53,153.23,144.98$, $139.15,137.80,133.92,133.50,131.48,130.55,129.97,128.35$, $127.93,127.34,127.16,122.95,112.54,71.55,65.91,54.28,43.21$, 25.31, 14.28, 13.47. HRMS (ESI): $m / z$ calcd for $\mathrm{C}_{27} \mathrm{H}_{30} \mathrm{NO}^{+}[\mathrm{M}]^{+}$ 384.2322 , found 384.3217 .

\subsection{Cell models}

MCF-7 (ER $\alpha+/$ PR+/HER2+), MDA-MB-231 (ER-/PR-/HER2-) cells were used to establish cisplatin cell models. The cells were treated with $25 \mu \mathrm{g} / \mathrm{mL}$ cisplatin individually for $24 \mathrm{~h}$. Carnosol cell models were established using MCF-7, MDA-MB-231 cells by treating with $40 \mathrm{mM}$ carnosol for $8 \mathrm{~h}$. Before imaging, the cells were pretreated with BCy-CYP for $30 \mathrm{~min}$ at $37^{\circ} \mathrm{C}$.

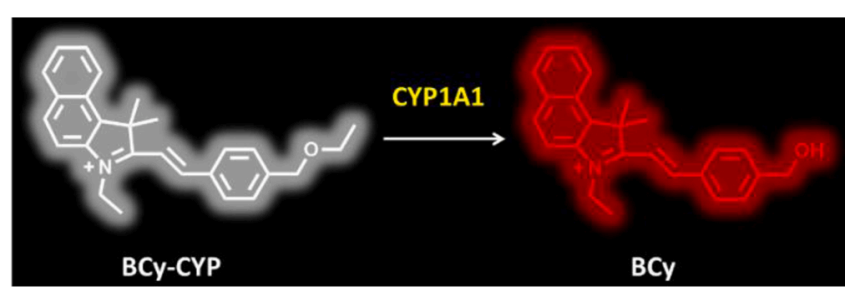

Scheme 1. Detection Mechanism of BCy-CYP against CYP1A1.

\subsection{In vivo imaging in living mice}

All the MCF-7 tumor model were administered an intratumorally injected of BCy-CYP (100 $\mu \mathrm{M}, 50 \mu \mathrm{l}$ in 1:99 DMSO/saline v/v). Eventually, all groups of mice were anesthetized by intraperitoneal (i.p.) injections of $4 \%$ chloral hydrate $(0.25 \mathrm{~mL})$. Mice images were taken by a Perkinelmer IVIS Lumina XRMS Series III In Vivo Imaging System, with an excitation filter of $550 \mathrm{~nm}$ and an emission of 600-700 $\mathrm{nm}$. The results were displayed as the mean standard deviation of 5 independent measurements. Besides, we merged the fluorescence image with the bright field image to clearly show the reaction site of the MCF-7 tumor mice. All mice experimental procedures were performed according to institutional regulations and guidelines for the use and care of laboratory animals and were approved by the Institutional Animal Care and Use Committee in Binzhou Medical University, Yantai. Approval Number: No. BZ2014-102R.

\subsection{Tissue preparation}

Mice were sacrificed by cervical dislocation at a fixed time of the day and their tumor were excised out immediately, washed in chilled physiological saline and blotted dry. Then the tumor tissue of mice model were prepared to frozen sections and stained with $5 \mu \mathrm{M} \mathrm{BCy-CYP}$ to confirm the amount of CYP1A1.

\section{Results and discussion}

\subsection{The design strategies of probes $B C y-C Y P$}

The synthetic approaches of BCy-CYP probe was outlined in Scheme S1. The synthetic details of compounds were shown in the Supporting Information. The mechanism for the probes was shown in Scheme 1. All the compound were characterized by ${ }^{1} \mathrm{H}$ NMR, ${ }^{13} \mathrm{C}$ NMR and HRMS. A benzoindocyanine dye ( $\mathrm{BCy}$ ), as the fluorophore unit, was applied for emitting the signal, which with the emission wavelength was located in $630 \mathrm{~nm}$. In this work, we incorporated the specific recognition moiety of the ethoxy group into fluorophore BCy to obtain the probe BCy-CYP for detection of CYP1A1. CYP1A1 has the dealkylation for $O$-alkyl groups [30], therefore the ethoxy group as response unit of CYP1A1 has specific response and high sensitivity [24]. Ethoxy group in BCy-CYP is directly weakens the fluorescence intensity of BCy; therefore, BCy-CYP exhibits weak fluorescence. Then CYP1A1 recognizes the ethoxy in BD-AChE, the ether bond is cleaved, and BCy-CYP is transformed to free BCy with fluorescence enhancement. The examination mechanism was outlined in Scheme 1. We would real-time monitoring CYP1A1 and providing a direct and significant evidence on the correlation between the CYP1A1 and breast cancer by using BCy-CYP probe. Furthermore, inhibition of CYP1A1 by carnosol efficiently induced cell apoptosis in breast cancer. And the synergy of carnosol and cisplatin induced more effectiveness therapeutic than that treated with cisplatin alone.

\subsection{Spectroscopic properties}

Under imitated physiological conditions (10 mM HEPES buffer, $\mathrm{pH}$ 7.4), the absorption and fluorescence spectra of BCy-CYP $(10 \mu \mathrm{M})$ were 

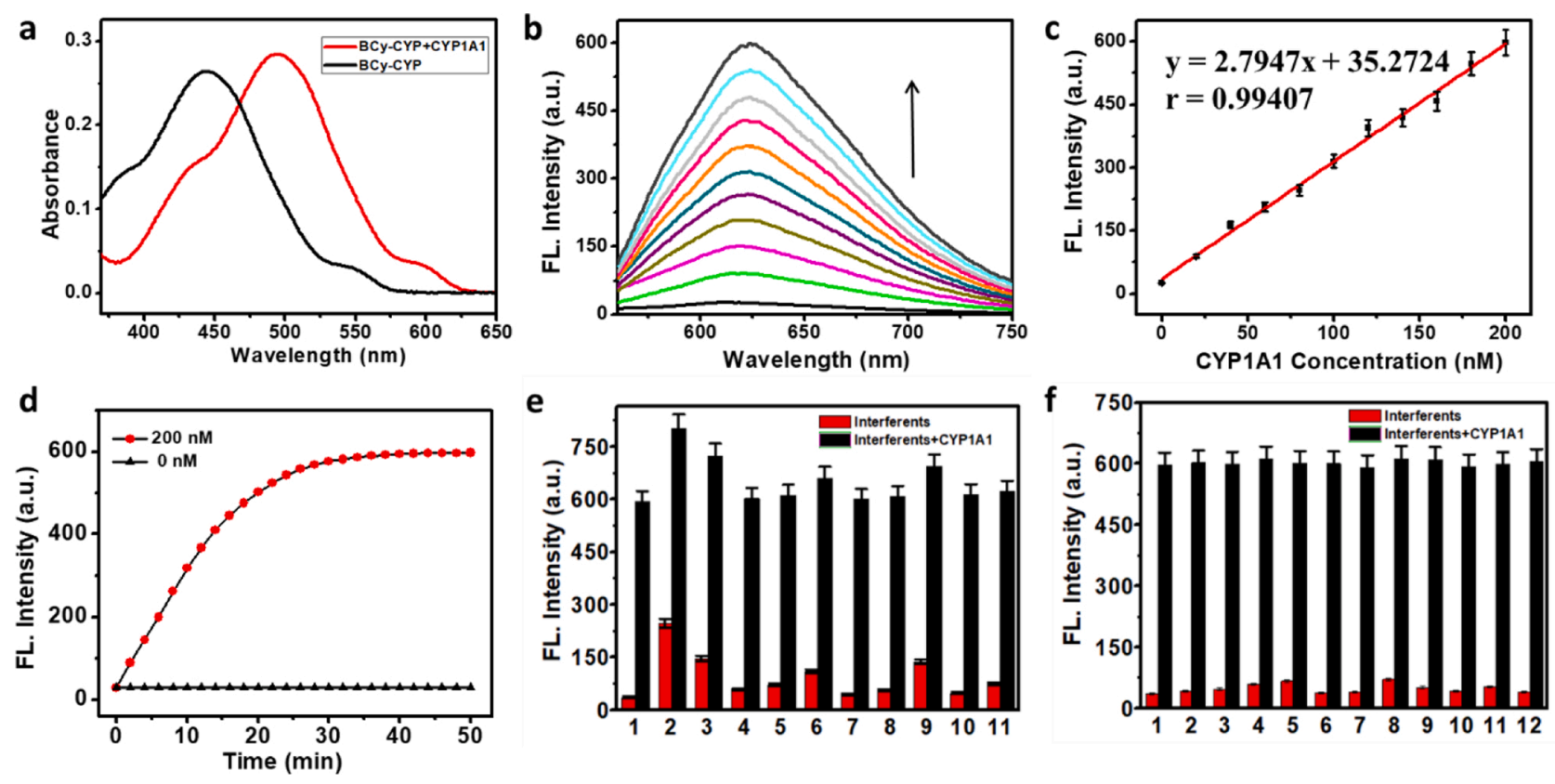

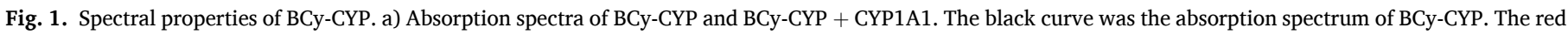

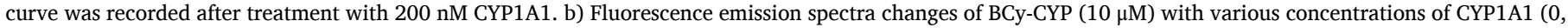

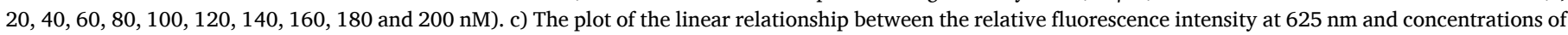

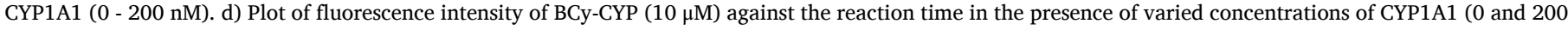

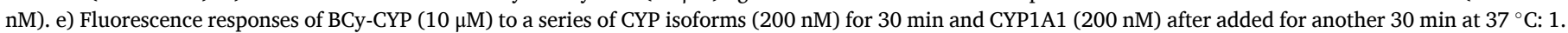

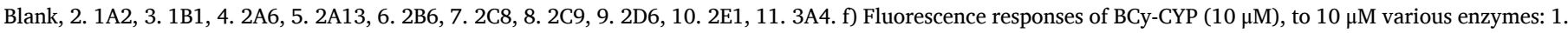

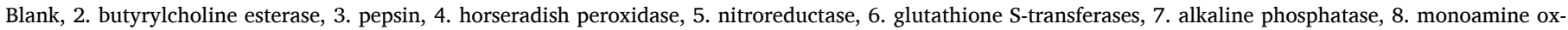

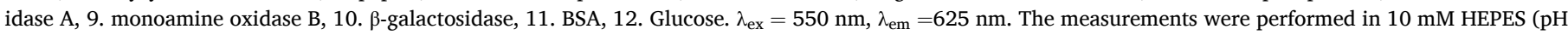
7.4) at $37^{\circ} \mathrm{C}$. (For interpretation of the references to colour in the Figure, the reader is referred to the web version of this article).

detected. The results presented in Fig. 1a, the maximum absorption wavelength of BCy-CYP was at $440 \mathrm{~nm}$. In the presence of $200 \mathrm{nM}$ CYP1A1, the maximum absorption peak shifted to $500 \mathrm{~nm}$. Under the condition of CYP1A1 concentration range of $0-200 \mathrm{nM}$, ВСу-CYP fluorescence titration was determined. As presented in Fig. $1 \mathrm{~b}$, the relevance fluorescence emission profiles increased with the peaks centered at $625 \mathrm{~nm}$. The excitation wavelength of probe was $500 \mathrm{~nm}$. The linear concentration-dependent fluorescence response range between BCy-CYP and CYP1A1 was 0-200 nM (Fig. 1c). The equation obtained from the calibration curve was $\mathrm{F}_{625 \mathrm{~nm}}=2.7947$ [CYP1A1] nM $+35.2724(\mathrm{r}=0.9941)$. Under the experimental conditions, the detection limit of BCy-CYP toward CYP1A1 was calculated to be $2.7 \mathrm{nM}$ $(3 \sigma / \mathrm{k})$, suggesting sensitivity highly sensitive of BCy-CYP probe towards CYP1A1 examination. These results investigated that BCy-CYP probe could be available for qualitatively and quantitatively test of CYP1A1.

\subsection{Kinetic investigations of BCy-CYP toward CYP1A1}

With the synthetic probe, we then evaluated BCy-CYP toward CYP1A1 under imitated physiological conditions (10 mM HEPES buffer, $\mathrm{pH}$ 7.4). As illustrated in Fig. 1d, we assessed the reaction activity of CYP1A1 towards BCy-CYP. BCy-CYP fluorescence kinetic curves with different concentrations of CYP1A1 $(0,200 \mathrm{nM})$ was studied within 30 min. It is indicates that CYP1A1 higher concentration emerged a faster reaction and a larger fluorescence enhancement. The fluorescence signal could reach the plateau within 25 min with the CYP1A1 concentration was at $200 \mathrm{nM}$. These results suggest that BCy-CYP displayed a very quick response to CYP1A1. The rapid response behavior induces BCyCYP probe a unique capability for real-time bioimaging of intracellular CYP1A1.

\subsection{Selectivity of BCy-CYP towards CYP1A1}

We next examined the fluorescence response of BCy-CYP toward a series of CYP isoforms to evaluate the selective of BCy-CYP. As shown in Fig. 1e, a remarkable change in the fluorescence intensity among all the detected CYP isoforms after added CYP1A1. After added CYP1A1, compared to CYP1A2, CYP1B1, CYP2B6, CYP2D6 and other CYPs (include 1B1, 2A6, 2A13, 2B6, 2C8, 2C9, 2D6, 2E1 and 3A4), there was $\sim 2.5$-fold, $\sim 4$-fold, $\sim 5$-fold, $\sim 4$-fold and $\sim 10$-fold difference in fluorescence intensity for CYP1A1, respectively. Furthermore, as presented in Fig. 1f, BCy-CYP probe was incubated with various types of enzymes (butyrylcholine esterase, pepsin, horseradish peroxidase, nitroreductase, glutathione $S$-transferases, alkaline phosphatase, monoamine oxidase A, monoamine oxidase B, $\beta$-galactosidase), BSA and glucose. The results certainly indicated that only added CYP1A1, a remarkable increase of fluorescence emission could be observed. In conclusion, these results revealed that $\mathrm{BCy}-\mathrm{CYP}$ was suitable for the sensitive detection of CYP1A1 with excellent selectivity, and the excellent responsiveness of $\mathrm{BCy}$-CYP towards CYP1A1 was not affected common biological various enzymes exist in cells or tissues.

\subsection{Imaging of CYP1A1 in living cells}

Since BCy-CYP probe exhibited greater selectivity towards CYP1A1, we then explored the application of BCy-CYP probe for test of CYP1A1 in living cells. The breast cancer cells utilized in this study included MCF-7 and MDA-MB-231 cells with MCF-10A (normal breast epithelial cell line) as control. MTT assays was performed to investigate the cytotoxicity of different concentrations (from 0 to $100 \mu \mathrm{M}$ ) of BCy-CYP probe in MCF-7 cells and MDA-MB-231 cells. The results fully revealed that BCy-CYP probe exhibited low cytotoxicity to MCF-7 and MDA-MB-231 living cells (Figs. S3 and S4).

All the experimental cells were incubated with BCy-CYP at $37^{\circ} \mathrm{C}$, the 

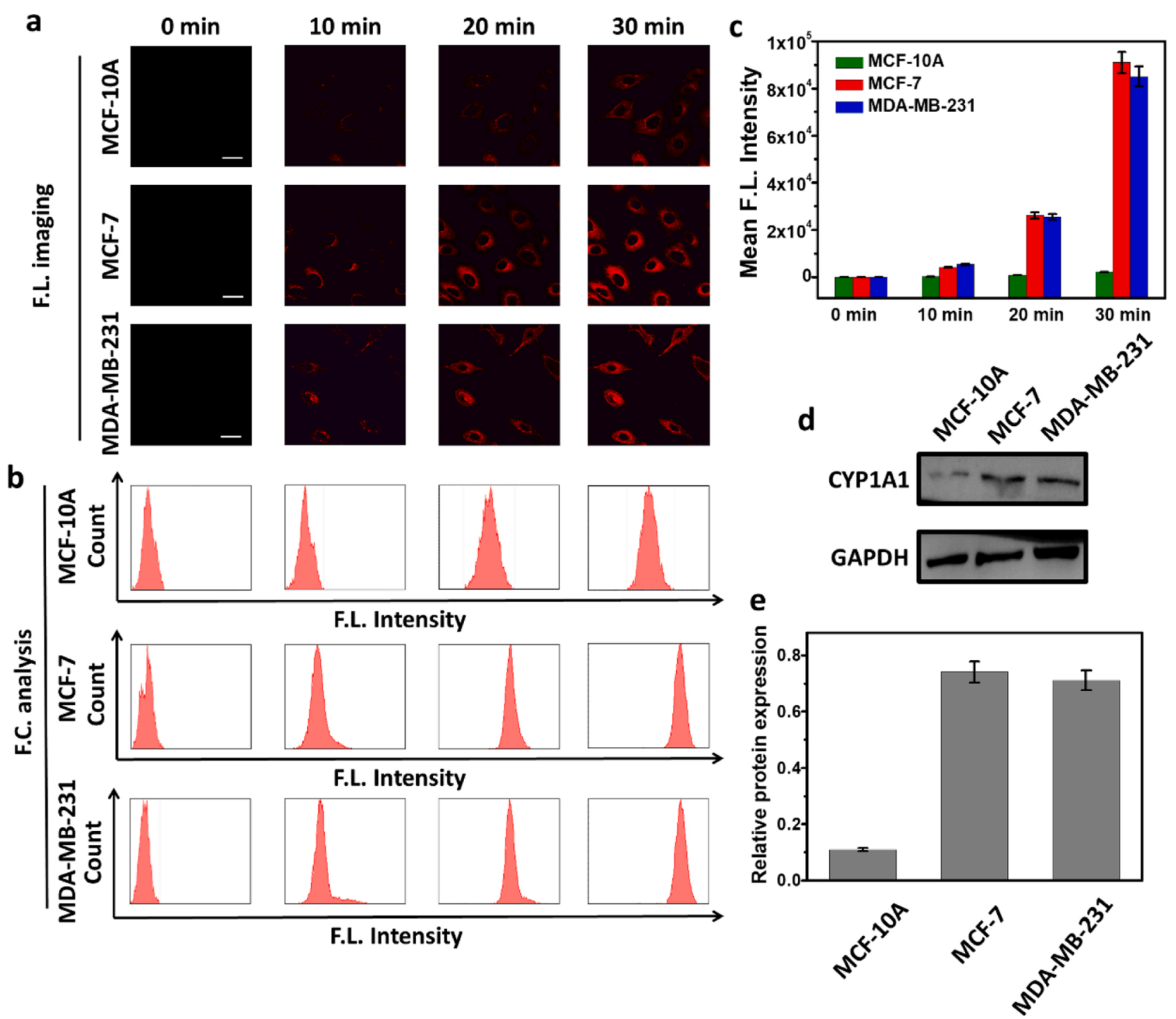

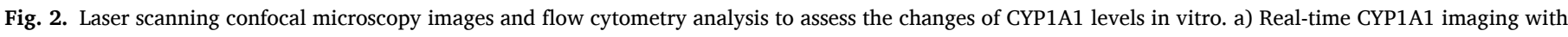

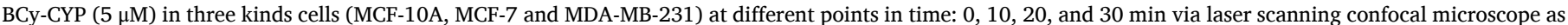

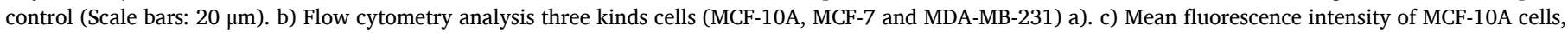

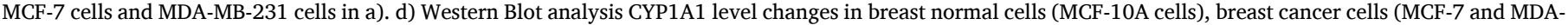

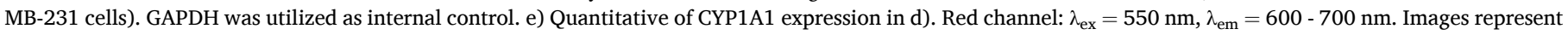
$\mathrm{n}=5$ independent experiments. (For interpretation of the references to colour in the Figure, the reader is referred to the web version of this article).

fluorescence changes were quantified by flow cytometry, and imaging by laser scanning confocal microscopy. The fluorescence of three types of cells in Fig. 2a gradually increased in the $30 \mathrm{~min}$ experimental process. The fluorescence of two kinds of breast cancer cells was significantly strong compared to MCF-10A cells, investigating the higher CYP1A1 level in human breast cancer cells. The bright-field images of Fig. 2a were presented in Fig. S5. Flow cytometry assay was further carried out to clarify the above experimental results (Fig. 2b). The average fluorescence intensity of three types of cells was displayed in Fig. 2c. Additional, the changes of CYP1A1 level was clarified by western blot assay. The results obtained from western blot analysis were consistent with the fluorescence images results and flow cytometry assay (Fig. 2d and e). These experiments disclosed that CYP1A1 level in breast cancer cells are higher than that in breast normal cells, and the probe $\mathrm{BCy}$-CYP could be utilized as a facilitative and effective tool to directly detect CYP1A1 level.

\subsection{Inhibition of CYP1A1 increases apoptosis}

To further analysis the relationship between CYP1A1 and breast cancer, we employed a pharmacologic method to decrease CYP1A1 levels. In general, cisplatin and other platinum-based compounds are considered as cytotoxic drugs which kill cancer cells by damaging DNA, inhibiting DNA synthesis and mitosis, and inducing apoptotic cell death. Drug metabolism is known to be catalyzed by a range of drugmetabolizing enzymes including the cytochromes P450 (CYPs) [31]. The CYP1A1 have the ability to catalyze the oxidation of foreign chemicals, including a wide variety of anticancer drugs [32]. Carnosol inhibits the AhR which regulates the basal and inducible expression level of CYP1 family members, including CYP1A1 [33]. Previous studies have been demonstrated that carnosol reduce CYP1A1 level in prostate cancer lines and in premalignant bronchial and tongue lines [34,35]. We next investigated the effects on two different breast cancer cells after using carnosol to lower the CYP1A1 level. Before imaging, all the experimental cells were incubated with BCy-CYP for $30 \mathrm{~min}$ at $37{ }^{\circ} \mathrm{C}$. 

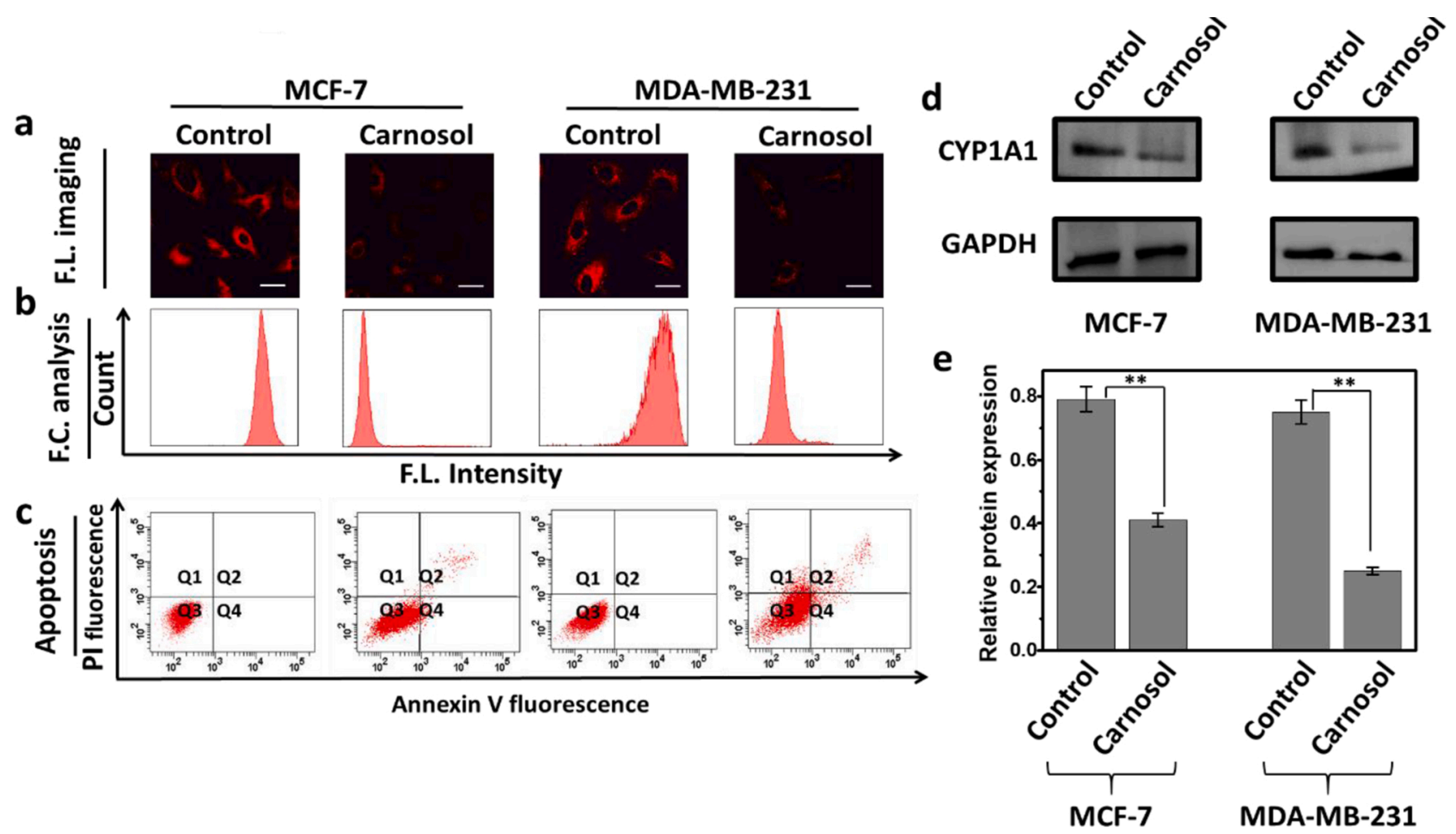

Fig. 3. The changes of CYP1A1 levels in MCF-7 and MDA-MB-231 cells. a) MCF-7 and MDA-MB-231 cells were pretreated with $40 \mathrm{mM}$ carnosol for $8 \mathrm{~h}$, respectively. Then confocal microscopy images of CYP1A1 level with BCy-CYP (5 $\mu \mathrm{M})$ in cells for 30 min (Scale bars: $20 \mu \mathrm{m})$. b) Flow cytometry analysis of a). c) The apoptosis rate of MCF-7 and MDA-MB-231 cells were analysed with or without carnosol. d) Western blot analysis CYP1A1 changes in MCF-7 and MDA-MB-231 cells with or without carnosol. GAPDH was utilized as internal control. e) Quantitative of CYP1A1 expression in d). Statistical analysis was performed using Student's t-test: ** $\mathrm{p}<0.01$. Red channel: $\lambda_{\mathrm{ex}}=550 \mathrm{~nm}, \lambda_{\mathrm{em}}=600-700 \mathrm{~nm}$. Data are presented as mean $\pm \mathrm{SD}(\mathrm{n}=5)$. (For interpretation of the references to colour in the Figure, the reader is referred to the web version of this article).

The Annexin V/PI assay was performed to evaluate the apoptotic rate. MCF-7 and MDA-MB-231 cells were pretreated with $40 \mathrm{mM}$ carnosol for $8 \mathrm{~h}$ in order to lower the CYP1A1 level before imaging. The fluorescence intensity in Fig. 3a decreases with the addition of carnosol, illustrating that CYP1A1 level in cells decreased. The results received from flow cytometry analysis in Fig. 3b showed the satisfactory consistency. The mean fluorescence intensity of two types of cells was displayed in Fig. 3d. Furthermore, we carried out western blot analysis to clarify the variation of CYP1A1 level, which further revealed the effective suppression of carnosol to CYP1A1 (Fig. 3e and f). As shown in Fig. 3c, after adding carnosol, the apoptosis rate of breast cancer cells increased, validating that carnosol might had a good therapeutic effect for breast cancer. Our results fully indicated that inhibition of CYP1A1 efficiently induced apoptosis in breast cancer cells. Therefore, the inhibition of CYP1A1 by carnosol was beneficial to the relief of breast cancer.

\subsection{A new synergistic therapeutic strategy for breast cancer}

Encouraged by the experimental results that inhibition of CYP1A1 efficiently increased apoptosis rate of breast cancer cells, we next devoted efforts to investigate the adjunctive therapeutic effect of inhibition of CYP1A1 by carnosol, and synergistic therapeutic effect of chemotherapy drug with carnosol. Cisplatin and its derivatives are vital drugs in the treatment for a wide range of human cancers [36], including breast cancer, testicular cancer, neck and head cancer, and others [37]. Thus, we chose cisplatin as an effective treatment for breast cancer. As indicated in Fig. 4a, the two different breast cancer cells were respectively divided into four groups. The first groups were selected as the control. The second cell groups were added $25 \mu \mathrm{g} / \mathrm{mL}$ cisplatin individually for $24 \mathrm{~h}$ before imaging. The third cell groups were treated with $40 \mathrm{mM}$ carnosol for $8 \mathrm{~h}$ before imaging. The cells in the fourth groups were co-treated with $40 \mathrm{mM}$ carnosol for $8 \mathrm{~h}$ and $25 \mu \mathrm{g} / \mathrm{mL}$ cisplatin for
$24 \mathrm{~h}$ as introduced in the above groups. As expected, the results of fluorescence intensity could be displayed in an order: control group $>$ cisplatin group $>$ carnosol group $>$ carnosol + cisplatin group (Fig. 4a). These results revealed the fluctuation of CYP1A1 levels and the CYP1A1 levels in carnosol + cisplatin group drops the most. The level of CYP1A1 is slightly reduced after adding cisplatin, which may be caused by a decrease in the number of living cells. The fluctuation of CYP1A1 levels in the four groups was also verified by western blot (Fig. $4 \mathrm{~d}$ and $4 \mathrm{e}$ ). We further employed flow cytometry assay to test the changes of fluorescence intensity (Fig. 4b). Besides, flow cytometry was applied to check the apoptosis rate of cells (Fig. 4c). The apoptosis rate of synergetic carnosol and cisplatin were monitored with significant increase than carnosol or cisplatin alone for two types of breast cancer cells, certifying that the synergistic therapy of carnosol and cisplatin displayed more effective therapeutic than only treated with carnosol or cisplatin alone. These results implied that the inhibition of CYP1A1 might consider being a novel synergistic therapeutic method for breast cancer.

\subsection{Imaging of CYP1A1 in tumor-bearing mice}

Due to our probe BCy-CYP were displayed to be efficacious in living cells, we next studied its efficiency in tumor-bearing mice. The application of BCy-CYP for imaging in vivo was investigated in MCF-7 tumorbearing mice. Four groups of tumor-bearing mice were chosen for carrying out the therapeutic test: in the first group, the tumor-bearing mice injected via tail vein with saline as the control. The second group were orally administered a $30 \mathrm{mg} / \mathrm{kg}$ carnosol once a day for 4 weeks. In the third group, cisplatin $(0.5 \mathrm{mg} / \mathrm{ml} \times 0.5 \mathrm{~mL})$ was given by intraperitoneal injection every other day for 4 weeks. The fourth group was administrated with carnosol and cisplatin as described in the second and third groups. We first measured the CYP1A1 level in vivo and ex vivo with BCy-CYP. After intratumorally injected BCy-CYP for $1 \mathrm{~h}$, the tumor- 

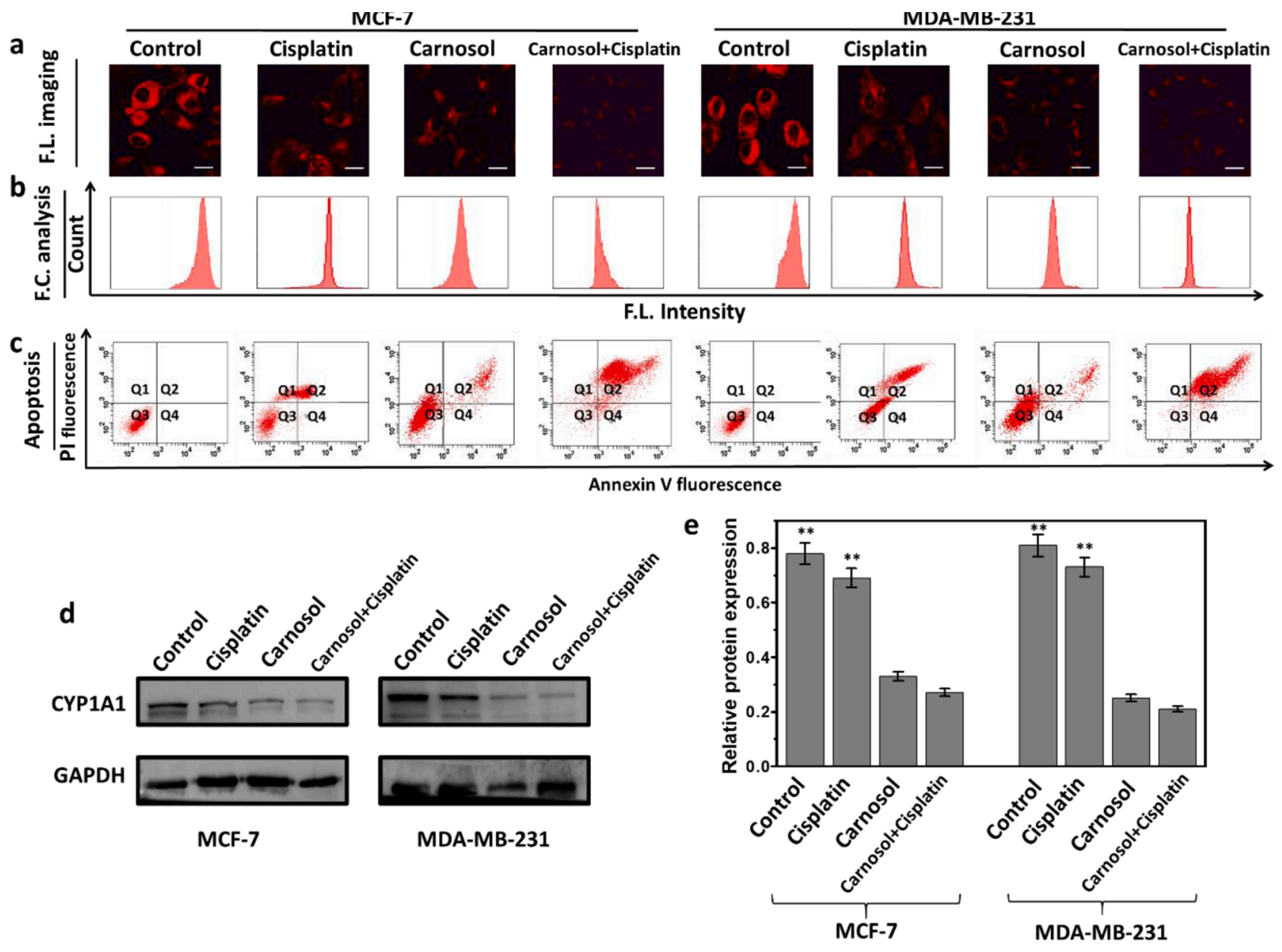

Fig. 4. The changes of CYP1A1 levels in MCF-7 and MDA-MB-231 cells. a) Confocal microscopy images of CYP1A1 level with BCy-CYP (5 $\mu$ M) in MCF-7 and MDAMB-231 cells for $30 \mathrm{~min}$. The first cell group were untreated. The second group were treated with $25 \mu \mathrm{g} / \mathrm{mL}$ cisplatin individually for $24 \mathrm{~h}$ before imaging. The third cell groups were treated with $40 \mathrm{mM}$ carnosol for $8 \mathrm{~h}$ before imaging. The fourth groups were co-treated with $40 \mathrm{mM}$ carnosol for $8 \mathrm{~h}$ and $25 \mu \mathrm{g} / \mathrm{mL}$ cisplatin for $24 \mathrm{~h}$, respectively (Scale bars: $20 \mu \mathrm{m}$ ). b) Flow cytometry analysis of a). c) Apoptosis analysis of two kinds of breast cancer cells as introduced in a). d) Western blot analysis CYP1A1 changes in MCF-7 and MDA-MB-231 cells. GAPDH was utilized as internal control. e) Quantitative of CYP1A1 expression in d). Statistical analysis was performed using Student's t-test: **p $<0.01$. Red channel: $\lambda_{\mathrm{ex}}=550 \mathrm{~nm}, \lambda_{\mathrm{em}}=600-700 \mathrm{~nm}$. Data are presented as mean \pm SD $(\mathrm{n}=5$ ). (For interpretation of the references to colour in the Figure, the reader is referred to the web version of this article).

bearing mice were subjected to in vivo imaging system for CYP1A1 detection. Under different treatment conditions, the fluorescence intensities were gathered in the order: control group $>$ cisplatin group $>$ carnosol group $>$ carnosol + cisplatin group (Fig. 5a). The variation of the CYP1A1 levels in tumors were as expected, which revealed the effective inhibition of carnosol to CYP1A1 in mice. The mean fluorescence intensity of Fig. 5a was displayed in Fig. 5e. Fluorescence imaging of the isolated tumors and tumor slices was performed in vitro, which corresponded to the imaging results of mice in vivo (Fig. 5b and d). We carried out H\&E pathological staining to clarify the tumor tissue (Fig. 5c). These above results revealed that the probe BCy-CYP had an outstanding ability to detect CYP1A1 levels in vivo. The probe had a significant potential value for clinical auxiliary diagnosis of breast cancer. Additionally, the tumors sizes and body weights of tumorbearing mice were measured every two days during the 16 day period. As indicated in Fig. 5f, $g$ and h, compared with carnosol or cisplatin alone, the synergistic therapy of carnosol and cisplatin displayed better inhibitory effects on tumor growth, disclosing that the synergistic therapy of carnosol and cisplatin had greater therapeutic effects. Therefore, in terms of overall therapeutic effect, inhibition of CYP1A1 by carnosol and cisplatin would be given priority as one of the adjuvant treatments for breast cancer.

\section{Conclusion}

In this study,we synthesized a fluorescent BCy-CYP probe to detect the changes of CYP1A1 level in breast normal and cancer cells, and in tumor-bearing mice. Through the using of BCy-CYP, we verify that the intracellular CYP1A1 plays crucial roles in breast cancer. Our experimental results certify that the CYP1A1 level in breast cancer cells are higher than in normal cells, which provide the basis for using BCy-CYP to detect cancerous tissue and normal tissue. To explore the influence of changes in CYP1A1 level on the treatment of breast cancer, we further confirm that inhibition of CYP1A1 by carnosol efficiently induced apoptosis in breast cancer cells. And we disclose that the synergistic therapy of carnosol and chemotherapy drug cisplatin display more effective therapeutic than treatment with only carnosol or chemotherapy drug. BCy-CYP probe is a new chemical tools to accurately diagnose breast cancer. CYP1A1 may be a useful indicator for early detection of breast cancer.

\section{CRediT authorship contribution statement}

Yan Huang: Conceptualization, Validation, Formal analysis, Investigation, Writing - original draft. Lei Yu: Data curation, Methodology, Investigation, Software. Pengpeng Lu: Conceptualization, Software, 

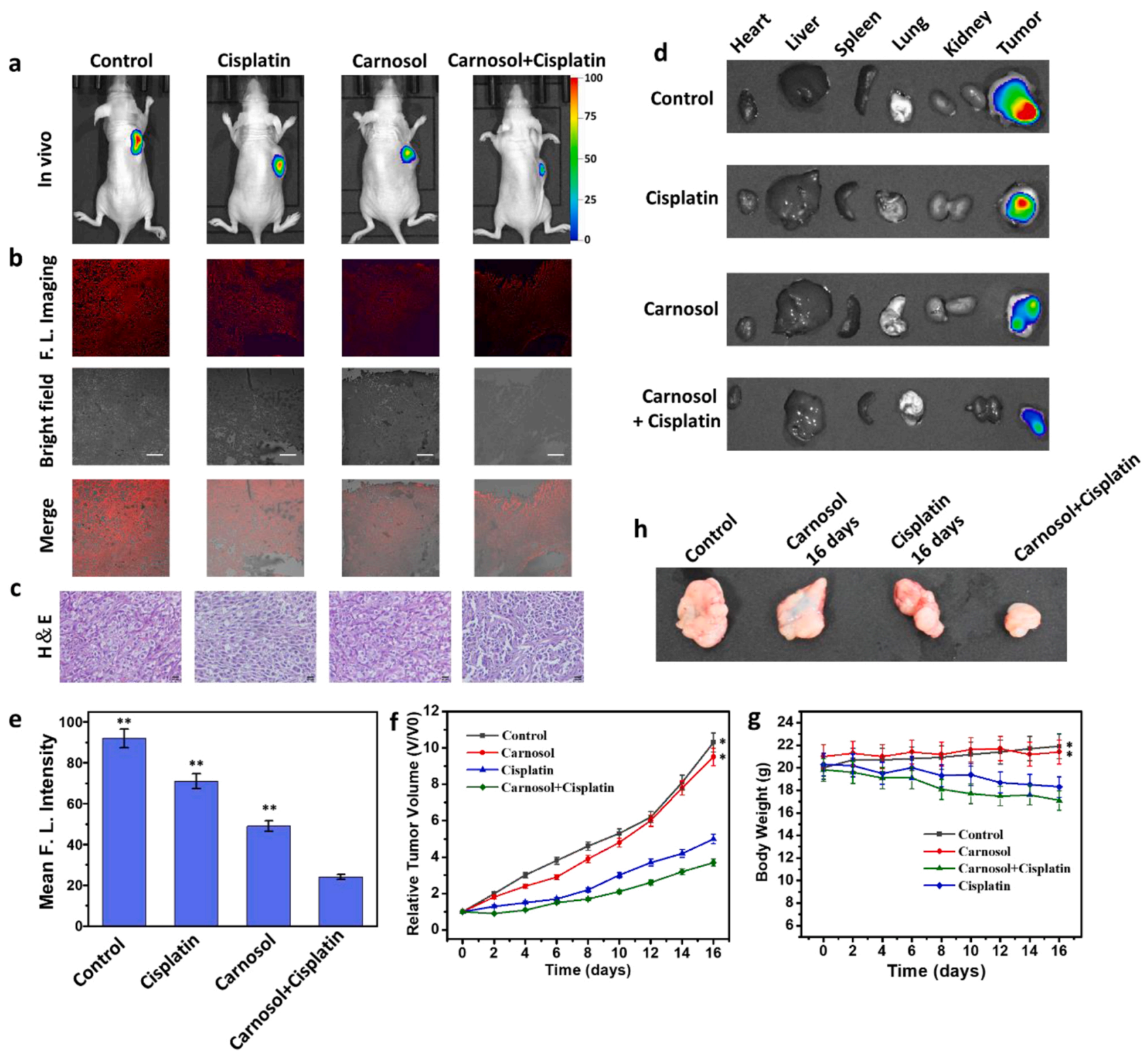

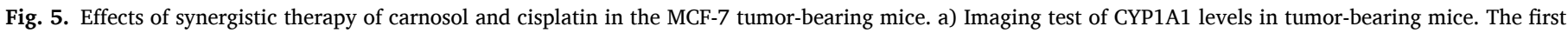

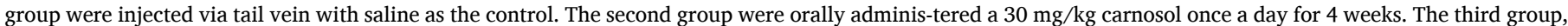

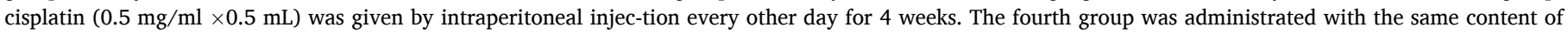

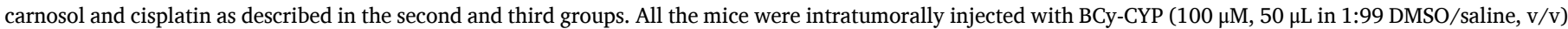

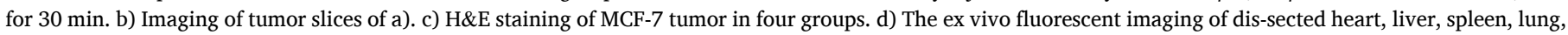

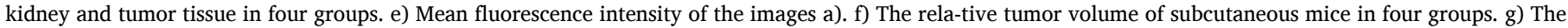

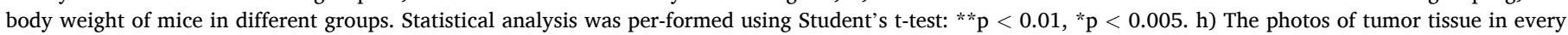
groups. Fluorescence imaging channel: $\lambda \mathrm{ex}=550 \mathrm{~nm}, \lambda \mathrm{em}=600-700 \mathrm{~nm}$. Data are presented as mean $\pm \mathrm{SD}(\mathrm{n}=5)$.

Visualization, Writing - review \& editing. Yinghui Wei: Validation, Formal analysis, Investigation, Validation. Xiaoyan Wang: Conceptualization, Funding acquisition, Methodology, Writing - review \& editing. Lingxin Chen: Funding acquisition, Conceptualization, Methodology, Writing - review \& editing.

\section{Declaration of Competing Interest}

The authors declare no competing financial interest.

\section{Acknowledgment}

This work was supported by the National Nature Science Foundation of China (Nos. 81573393, 21804010, 21976209, 22007005), the science and technology innovation development plan of Yantai of China (No. 2020MSGY113, 2020YT06000898), the Research Initiation Fund of Binzhou Medical University (Grant No. BY2019KYQD39, BY2020KYQD01), Taishan Scholar Project Special Funding (No. ts20190962), Shandong Peninsula Engineering Research Center of Comprehensive Brine Utilization (2018LS014), Weifang Science and technology development project (2019GX076).

\section{Appendix A. Supplementary data}

Supplementary material related to this article can be found, in the online version, at doi:https://doi.org/10.1016/j.snb.2021.130233. 


\section{References}

[1] J. Ferlay, M. Colombet, I. Soerjomataram, C. Mathers, D.M. Parkin, M. Pineros, A. Znaor, F. Bray, Estimating the global cancer incidence and mortality in 2018: GLOBOCAN sources and methods, Int. J. Cancer 8 (2019) 1941-1953.

[2] B.L. Eckhardt, P.A. Francis, B.S. Parker, R.L. Anderson, Strategies for the discovery and development of therapies for metastatic breast cancer, Nat. Rev. Drug Discov. 6 (2012) 479-497.

[3] A.A. Onitilo, J.M. Engel, R.T. Greenlee, B.N. Mukesh, Breast cancer subtypes based on ER/PR and Her2 expression: comparison of clinicopathologic features and survival, Clin. Med. Res. 1-2 (2009) 4-13.

[4] M. Dowsett, L.A. Martin, I. Smith, S. Johnston, Mechanisms of resistance to aromatase inhibitors, J. Steroid Biochem. Mol. Biol. 1-5 (2005) 167-172.

[5] V.P. Androutsopoulos, A.M. Tsatsakis, D.A. Spandidos, Cytochrome P450 CYP1A1: wider roles in cancer progression and prevention, BMC Cancer (2009) 187.

[6] G.I. Murray, S. Patimalla, K.N. Stewart, I.D. Miller, S.D. Heys, Profiling the expression of cytochrome P450 in breast cancer, Histopathology 2 (2010) 202-211.

[7] G. Vinothini, S. Nagini, Correlation of xenobiotic-metabolizing enzymes, oxidativ stress and NFkappaB signaling with histological grade and menopausal status in patients with adenocarcinoma of the breast, Clin. Chim. Acta 5-6 (2010) 368-374.

[8] E. Brantley, V. Trapani, M.C. Alley, C.D. Hose, T.D. Bradshaw, M.F. Stevens, E. A. Sausville, S.F. Stinson, Fluorinated 2-(4-amino-3-methylphenyl)benzothiazoles induce CYP1A1 expression, become metabolized, and bind to macromolecules in sensitive human cancer cells, Drug Metab. Dispos. 12 (2004) 1392-1401.

[9] V. Trapani, V. Patel, C.O. Leong, H.P. Ciolino, G.C. Yeh, C. Hose, J.B. Trepel, M. F. Stevens, E.A. Sausville, A.I. Loaiza-Perez, DNA damage and cell cycle arrest induced by 2-(4-amino-3-methylphenyl)-5-fluorobenzothiazole (5F 203, NSC 703786) is attenuated in aryl hydrocarbon receptor deficient MCF-7 cells, Br. J. Cancer 4 (2003) 599-605.

[10] E. Caron, N. Rioux, O. Nicolas, H. Lebel-Talbot, B.A. Hamelin, Quantification of the expression and inducibility of 12 rat cytochrome $\mathrm{P} 450$ isoforms by quantitative RTPCR, J. Biochem. Mol. Toxicol. 6 (2005) 368-378.

[11] C. Rodriguez-Antona, R. Jover, M.J. Gomez-Lechon, J.V. Castell, Quantitative RTPCR measurement of human cytochrome P-450s: application to drug induction studies, Arch. Biochem. Biophys. 1 (2000) 109-116.

[12] V. Marx, Probes: seeing in the near infrared, Nat. Methods 7 (2014) 717-720.

[13] Y. Yang, Q. Zhao, W. Feng, F. Li, Luminescent chemodosimeters for bioimaging, Chem. Rev. 1 (2013) 192-270.

[14] X. Li, X. Gao, W. Shi, H. Ma, Design strategies for water-soluble small molecular chromogenic and fluorogenic probes, Chem. Rev. 1 (2014) 590-659.

[15] X. Chen, Y. Zhou, X. Peng, J. Yoon, Fluorescent and colorimetric probes for detection of thiols, Chem. Soc. Rev. 6 (2010) 2120-2135.

[16] J. Zhou, H. Ma, Design principles of spectroscopic probes for biological applications, Chem. Sci. 10 (2016) 6309-6315.

[17] M. Gao, F. Yu, C. Lv, J. Choo, L. Chen, Fluorescent chemical probes for accurate tumor diagnosis and targeting therapy, Chem. Soc. Rev. 8 (2017) 2237-2271.

[18] J. Mei, N.L. Leung, R.T. Kwok, J.W. Lam, B.Z. Tang, Aggregation-Induced Emission: Together We Shine, United We Soar!, Chem. Rev. 21 (2015) 11718-11940.

[19] D. Wu, A.C. Sedgwick, T. Gunnlaugsson, E.U. Akkaya, J. Yoon, T.D. James, Fluorescent chemosensors: the past, present and future, Chem. Soc. Rev. 23 (2017) $7105-7123$

[20] J. Fan, M. Hu, P. Zhan, X. Peng, Energy transfer cassettes based on organic fluorophores: construction and applications in ratiometric sensing, Chem. Soc. Rev. 1 (2013) 29-43.

[21] J.T. Hou, W.X. Ren, K. Li, J. Seo, A. Sharma, X.Q. Yu, J.S. Kim, Fluorescent bioimaging of pH: from design to applications, Chem. Soc. Rev. 8 (2017) 2076-2090.

[22] P. Verwilst, H.S. Kim, S. Kim, C. Kang, J.S. Kim, Shedding light on tau protein aggregation: the progress in developing highly selective fluorophores, Chem. Soc Rev. 7 (2018) 2249-2265.

[23] H.W. Liu, L. Chen, C. Xu, Z. Li, H. Zhang, X.B. Zhang, W. Tan, Recent progresses in small-molecule enzymatic fluorescent probes for cancer imaging, Chem. Soc. Rev. 18 (2018) 7140-7180.

[24] H. Ji, X. Zhang, Y. Dai, T. Xue, S. Misal, Z. Oi, A highly selective ratiometric fluorescent probe based on naphthalimide for detection and imaging of CYP1A1 in living cells and zebrafish, Analyst 24 (2019) 7390-7397.

[25] Z.R. Dai, L. Feng, Q. Jin, H. Cheng, Y. Li, J. Ning, Y. Yu, G.B. Ge, J.N. Cui, L. Yang, A practical strategy to design and develop an isoform-specific fluorescent probe fo a target enzyme: CYP1A1 as a case study, Chem. Sci. 4 (2017) 2795-2803.

[26] J. Ning, Z. Tian, B. Wang, G. Ge, Y. An, J. Hou, C. Wang, X. Zhao, Y. Li, X. Tian, Z. Yu, X. Huo, C. Sun, L. Feng, J. Cui, X. Ma, A highly sensitive and selective two- photon fluorescent probe for real-time sensing of cytochrome P450 1A1 in living systems, Mater. Chem. Front. (2018) 2013-2020.

[27] Z. Dai, G. Ge, L. Feng, J. Ning, L. Hu, Q. Jin, D. Wang, X. Lv, T. Dou, J. Cui, L. Yang, A highly selective ratiometric two-photon fluorescent probe for human cytochrome P450 1A, J. Am. Chem. Soc. (2015) 14488-14495.

[28] T. Xue, Y. Dai, X. Zhang, Y. Cheng, X. Gu, H. Ji, S. Misal, Z. Qi, Ultrasensitive nearinfrared fluorescent probe with large stokes shift for real-time tracing of CYP1A1 in living cells and zebrafish model, Sens. Actuators B Chem. (2019) 265-272.

[29] Q. Jin, H. Ma, L. Feng, P. Wang, R. He, J. Ning, L. Yang, G. Ge, Sensing cytochrome P450 1A1 activity by a resorufin-based isoform-specific fluorescent probe, Chinese Chem. Lett. (2020) 2945-2949.

[30] A.A. Walsh, G.D. Szklarz, E.E. Scott, Human cytochrome P450 1 A1 structure and utility in understanding drug and xenobiotic metabolism, J. Biol. Chem. 18 (2013) 12932-12943.

[31] S. Dasari, P.B. Tchounwou, Cisplatin in cancer therapy: molecular mechanisms of action, Eur. J. Pharmacol. (2014) 364-378.

[32] K. Fujita, Cytochrome P450 and anticancer drugs, Curr. Drug Metab. 1 (2006) 23-37.

[33] A. Mohebati, J.B. Guttenplan, A. Kochhar, Z.L. Zhao, W. Kosinska, K. Subbaramaiah, A.J. Dannenberg, Carnosol, a constituent of Zyflamend, inhibits aryl hydrocarbon receptor-mediated activation of CYP1A1 and CYP1B1 transcription and mutagenesis, Cancer Prev. Res. (Phila) 4 (2012) 593-602.

[34] J.J. Johnson, D.N. Syed, C.R. Heren, Y. Suh, V.M. Adhami, H. Mukhtar, Carnosol, a dietary diterpene, displays growth inhibitory effects in human prostate cancer PC3 cells leading to G2-phase cell cycle arrest and targets the 5'-AMP-activated protein kinase (AMPK) pathway, Pharm. Res. 9 (2008) 2125-2134.

[35] E.A. Offord, K. Mace, C. Ruffieux, A. Malnoe, A.M. Pfeifer, Rosemary components inhibit benzo[a]pyrene-induced genotoxicity in human bronchial cells, Carcinogenesis 9 (1995) 2057-2062.

[36] J. Ma, X. Hu, J. Li, D. Wu, Q. Lan, Q. Wang, S. Tian, W. Dong, Enhancing conventional chemotherapy drug cisplatin-induced anti-tumor effects on human gastric cancer cells both in vitro and in vivo by Thymoquinone targeting PTEN gene, Oncotarget 49 (2017) 85926-85939.

[37] F. Liang, S. Zhang, H. Xue, Q. Chen, Risk of second primary cancers in cancer patients treated with cisplatin: a systematic review and meta-analysis of randomized studies, BMC Cancer 1 (2017) 871.

Yan Huang is currently as a professor at School of Pharmacy, Binzhou Medical University. He received his pH.D., joint-educated, at Shandong Normal University, and Yantai Institute of Coastal Zone Research, Chinese Academy of Sciences, in 2019. His research in terests focus on functional fluorescent probe, theranostics, and functional nanomaterial.

Lei Yu was received his MSc degrees in 2010 from Shandong Normal University. He is studying for his doctoral degree in analytical chemistry at the same university. His current research interest is chemical sensors and biosensors.

Pengpeng Lu is currently a master candidate, joint-educated, at Binzhou Medical University, and Yantai Institute of Coastal Zone Research, Chinese Academy of Sciences, since 2019. His current interest in research focuses on fluorescence bioimaging.

Yinghui Wei is currently a master candidate, joint-educated, at Binzhou Medical University, and Yantai Institute of Coastal Zone Research, Chinese Academy of Sciences, since 2019. She received her BS from Binzhou Medical University, in 2019. Her current interest in research focuses on bioimaging.

Xiaoyan Wang is currently as an associate professor at School of Pharmacy, Binzhou Medical University. She received her pH.D., joint-educated, at Shandong Normal University, and Yantai Institute of Coastal Zone Research, Chinese Academy of Sciences, in 2017. Her research interests focus on the studies of functionalized nanoparticles for developing nanoscale biochemical analysis methods and molecular imprinting-based sample pretreatment technology.

Lingxin Chen has been a professor at Yantai Institute of Coastal Zone Research, Chinese Academy of Sciences, since 2009. He obtained his pH.D. in analytical chemistry at Dalian Institute of Chemical Physics, Chinese Academy of Sciences, in 2003. During 2004-2009, he worked at Department of Chemistry, Tsinghua University, and Department of Applied Chemistry, Hanyang University, respectively. His research interests include the studies of novel properties of materials such as functionalized nanoparticles \& functional probe molecules for developing nanoscale biochemical analysis methods and molecular imprinting-based sample pretreatment technology. 